\title{
Post-transplant Amputation Traumatic Neuroma of the Hilum and Extrahepatic Duct in a Liver Donor
}

\author{
Na Rae Kim · Hyun Yee Cho · Dong Hae Chung · Keon Kuk Kim ${ }^{1} \cdot$ Jae Hee Cho ${ }^{2}$ Seung Joon Choi ${ }^{3}$ \\ Departments of Pathology and ${ }^{1}$ General Surgery, ${ }^{2}$ Division of Gastroenterology, Department of Internal Medicine, \\ ${ }^{3}$ Department of Radiology, Gachon University Gil Medical Center, Incheon, Korea
}

Traumatic neuromas develop after injury to nerve fibers encased in Schwann cells. They occur mainly in the amputated stump of the extremities. ${ }^{1}$ Biliary traumatic neuroma was first described in 1928 by Husseinoff. $^{2}$ Since then, less than 100 cases of biliary traumatic neuroma have been reported. ${ }^{3,4}$ Most occur at the cystic duct stump after cholecystectomy. It may also occur in the main biliary tract following any injury, even liver transplantation.,

Herein, we report a case of post-transplant biliary traumatic neuroma in a donor after an 8-year interval. This rare biliary traumatic neuroma may be a risk factor for liver transplantationrelated biliary stricture.

\section{CASE REPORT}

A 53-year-old woman visited the emergency room for abruptly developed pruritus and jaundice. She had a past history of undergoing right hepatectomy and closure of left hepatic duct as a living donor liver transplantation (LDLT) to her husband 8 years previously. A computed tomography of the abdomen showed an enhancing lesion in eccentric wall thickening of the proximal bile duct, suggestive of hilar cholangiocarcinoma (Fig. 1A). The preoperative carbohydrate antigen 19-9 and carcinoembryonic antigen levels were within normal ranges. Liver function tests revealed total bilirubin at 1.5 (reference $<1.2 \mathrm{mg} / \mathrm{dL}$ ), gamma-glutamyl transferase at 258 (reference $<48 \mathrm{U} / \mathrm{L}$ ), alkaline phospha-

\section{Corresponding Author}

Dong Hae Chung, MD, PhD

Department of Pathology, Gachon University Gil Medical Center, 21 Namdong-daero

774beon-gil, Namdong-gu, Incheon 21565, Korea

Tel: +82-32-460-3866, Fax: +82-32-460-2394, E-mail: dhchung@gilhospital.com

Received: December 8, 2016 Revised: January 5, 2017

Accepted: January 19, 2017 tase at 177 (reference < $123 \mathrm{U} / \mathrm{L}$ ), and alanine transaminase at 49 (reference $<40$ ). Liver magnetic resonance images and cholangiopancreatography revealed an enhanced wall thickening in the common hepatic duct and dilated left proximal intrahepatic duct. An endoscopic ultrasonography revealed a mass-like heterogeneous echogenic lesion, measuring $15.6 \times 11.4 \mathrm{~mm}$, at the hilar level. A preoperative endoscopic retrograde cholangiopancreatography revealed the diameter of the proximal common bile duct dilated up to $9 \mathrm{~mm}$. A biopsy was done, and a biliary stent was placed to relieve the biliary obstruction. The preoperative biopsy showed only normal duodenal epithelium. At surgery, a firm adhesion to the adjacent portal vein and duodenal wall was found. She had a fibrotic and hard mass-like lesion in the hilum and proximal common bile duct. An intraoperative frozen biopsy of the proximal bile duct resection margin was done. A caudate lobectomy with bile duct resection and hepatojejunostomy was done under a presumptive diagnosis of cholangiocarcinoma. A periportal lymphadenectomy was done and a biopsy was taken from the portal vein.

Grossly, the received specimen showed the common bile duct external circumference dilated up to $2.2 \mathrm{~cm}$ (Fig. 1B). The inner surface of the resected bile duct was thickened and fibrotic, measuring $2.2 \times 1.8 \mathrm{~cm}$.

Under light microscopy, the serial sections of the thickened bile duct revealed hyperplastic and disorganized nerve fibers surrounded and dissected by thick collagenous fibrous connective tissue (Fig. 1C). The bile duct was compressed by fibrotic tissue. Small foci of mature lymphoid cells and hemosiderin-laden macrophages were also found. (Fig. 1D) Immunohistochemical stains were positive for S-100 protein (1:600, polyclonal, Dako, Glostrup, Denmark) and Masson-Trichrome stain revealed marked 

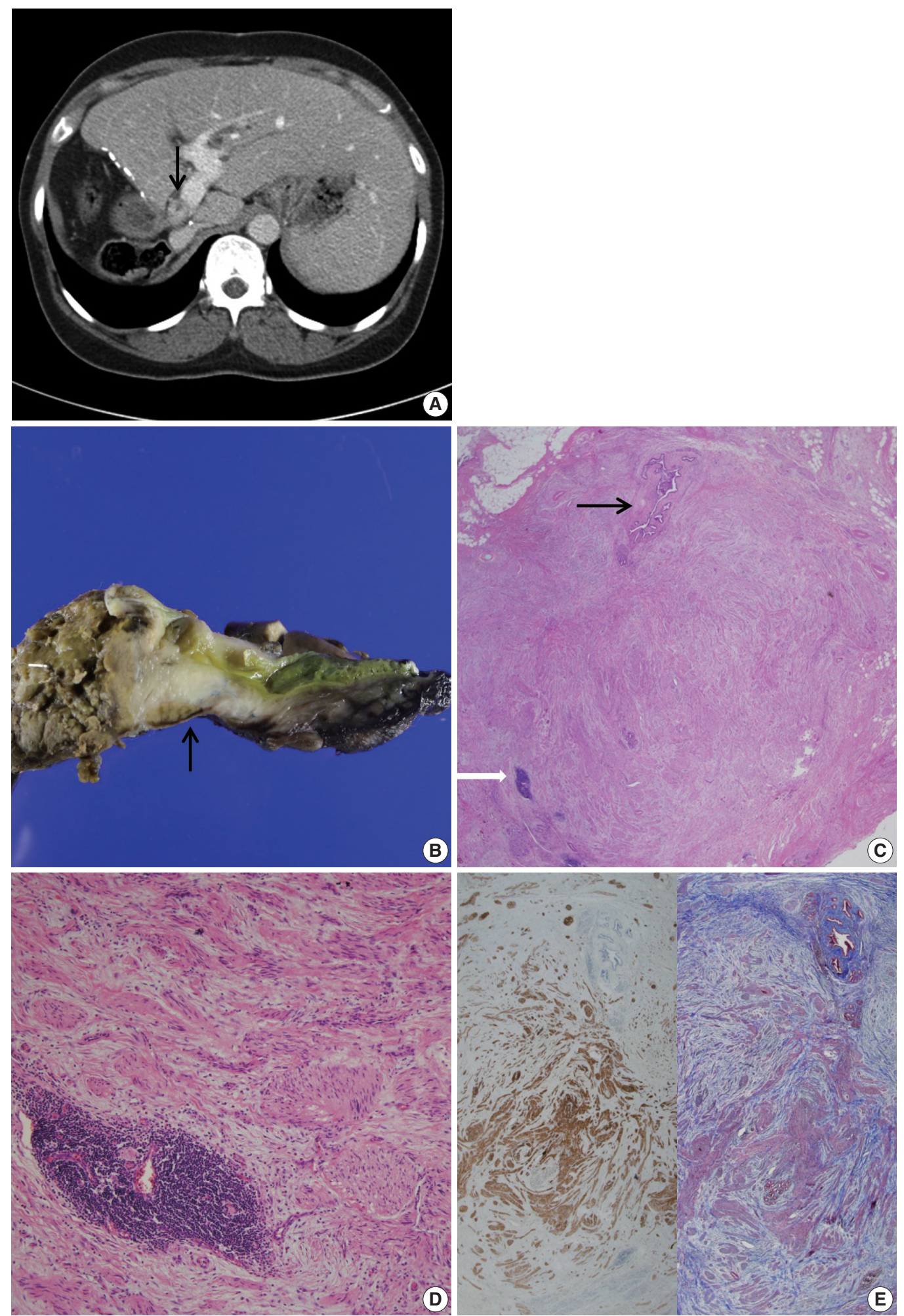

Fig. 1. (A) Abdominal computed tomography reveals an enhanced thickening (arrow) of the common bile duct wall. (B) Grossly, the resected common bile duct reveals a firm grayish mass-like lesion (arrow) at the proximal bile duct. (C) Microscopically, the thickened mass-like portion consists of abundant nerve fascicles mainly composed of axon fibers and Schwann cells, intermixed with collagen bundles. Note entrapped bile duct (black arrow) and focal lymphoid aggregates (white arrow). (D) Marked fibrosis and haphazard proliferation of nerve fascicles. Note lymphoid aggregates. (E) Marked collagen deposits are intervening in the perineurial and epineurial area of nerve fascicles (left: S-100 protein immunohistochemical stain, right: Masson-Trichrome stain). 
perineurial and epineurial fibrosis (Fig. 1E). The diagnosis was amputation traumatic neuroma of the bile duct. Proximal and distal bile duct resection margins were free of fibrosis. Nerve fascicles were seen up to the tunica media of the biopsied portal vein. Enlarged, up to $1.2 \mathrm{~cm}$, periportal lymph nodes showed reactive hyperplasia. On the fifth postoperative day, the serum bilirubin level was normalized. On the ninth postoperative day, the patient was discharged with an unremarkable course.

This study was approved by the Institutional Review Board of Gachon University Gil Hospital with a waiver of informed consent (GBIRB 2016-323).

\section{DISCUSSION}

LDLT was introduced in 1989, and the first Korean LDLT was done in 1994. ${ }^{7}$ According to the Korean Network of Organ Sharing, the number of adult LDLT (patients aged $>18$ years) has increased annually, with 911 cases of adult LDLT performed in Korea in 2015. This number represents $67.4 \%$ of total adult liver transplantation in Korea, whereas LDLT comprises less than $3 \%$ in the United States. Therefore, this dramatic difference in clinical practice in Korea raises concerns and doubts about the long term well-being of donors. ${ }^{8}$ The immediate postoperative complications on the donor side are infections, portal vein or inferior vena cava thrombosis, and remnant cystic duct causing so-called post-cholecystectomy syndrome. ${ }^{9}$ Studies on recipients' postoperative morbidity report that biliary stricture due to traumatic neuroma occurs only after duct-to-duct biliary anastomosis following liver transplantation. The first report of transplantationrelated biliary traumatic neuromas showed a $27.9 \%$ prevalence, ${ }^{5}$ but the risk of pathologically confirmed symptomatic biliary traumatic neuroma is about $0.5 \%{ }^{6}$

The biliary tract is richly innervated by the hepatic plexus, which is derived from the celiac plexus. Dissection of the biliary tract and dissected nerve fibers may induce inappropriate nerve regeneration, triggered by factors such as intraoperative thermal injury of the bile ducts and foreign bodies such as suture materials. ${ }^{4}$ Immunosuppressants of calcineurin inhibitors such as tacrolimus are also reported as another possible factor. ${ }^{10}$ However, not all sympathetic nerve fibers induce an amputation traumatic neuroma and the exact pathogenesis is still unknown.

Traumatic neuromas arising from bile duct after orthotopic liver transplantation have rarely been described. ${ }^{5,6}$ Previous articles have been focused on recipients of LDLT. However, the risk of biliary traumatic neuromas may be underestimated because they are usually asymptomatic even in severe stricture cases, like the present case. These asymptomatic period ranges from several months up to 45 years after the biliary operation. There are no specific radiologic findings of biliary traumatic neuroma, ${ }^{3}$ and the diagnosis of biliary traumatic neuroma is not made unless it is pathologically confirmed after resection. Pathologic findings are characterized by disorganized haphazard proliferating nerve fascicles growing out in various directions, resulting in a bulbshaped mass-like thickening, i.e., stump, resembling cholangiocarcinoma. ${ }^{4}$ Both end-to-end anastomosis and hepaticojejunostomy are most commonly advocated in symptomatic cases. Considering its possible pathogenesis, however, biliary traumatic neuromas can recur following the biliary operation.

Biliary traumatic neuromas represent a rare cause of biliary stricture in both donor and recipient after orthotopic liver transplantation. To avoid unnecessary wide resection, the physician should be aware of the possibility of biliary traumatic neuroma when facing unexplained anastomotic biliary stenosis, even when presenting with a mass-like lesion on radiology.

\section{Conflicts of Interest}

No potential conflict of interest relevant to this article was reported.

\section{REFERENCES}

1. Necmioglu S, Subasi M, Kayikci C, Young DB. Lower limb landmine injuries. Prosthet Orthot Int 2004; 28: 37-43.

2. Husseinoff $D$. Ueber einem Fall von Wucherung des Nervengewebes nach wiederholten Operationen der Gallenga: nge. Zbl Allg Path 1928; 43: 344-8.

3. Iannelli A, Fabiani P, Karimdjee BS, Converset S, Saint-Paul MC, Gugenheim J. Traumatic neuroma of the cystic duct with biliary obstruction: report of a case. Acta Gastroenterol Belg 2003; 66: 28-9.

4. Pickens A, Vickers SM, Brown KL, Reddy VV, Thompson JA. An unusual etiology of biliary hilar obstruction and the potential role of acidic fibroblast growth factor in the development of a biliary neuroma. Am Surg 1999; 65: 47-51.

5. Colina F, Garcia-Prats MD, Moreno E, et al. Amputation neuroma of the hepatic hilum after orthotopic liver transplantation. Histopathology 1994; 25: 151-7.

6. Navez J, Golse N, Bancel B, et al. Traumatic biliary neuroma after orthotopic liver transplantation: a possible cause of "unexplained" anastomotic biliary stricture. Clin Transplant 2016; 30: 1366-9.

7. Jeon H, Lee SG. Living donor liver transplantation. Curr Opin Organ Transplant 2010; 15: 283-7. 
8. Lee SG. Living-donor liver transplantation in adults. Br Med Bull 2010; 94: 33-48.

9. Ghobrial RM, Freise CE, Trotter JF, et al. Donor morbidity after living donation for liver transplantation. Gastroenterology 2008; 135: 468-
76.

10. Sosa I, Reyes O, Kuffler DP. Immunosuppressants: neuroprotection and promoting neurological recovery following peripheral nerve and spinal cord lesions. Exp Neurol 2005; 195: 7-15. 